\title{
Sexually Transmitted Infections in Pregnant Women: Integrating Screening and Treatment into Prenatal Care
}

\author{
Harold J. Lochner III' • Nizar F. Maraqa ${ }^{1}$
}

Published online: 21 August 2018

(c) Springer Nature Switzerland AG 2018

\begin{abstract}
Chlamydia, gonorrhea, and syphilis continue to be significant burdens to the health of pregnant women and their children despite easy and effective guidance for screening and treatment. Specific guidelines exist for screening for these infections as well as treatment and follow-up. In this article, we review the epidemiology of chlamydia, gonorrhea, and syphilis. Testing methodology is also reviewed as molecular-based techniques have replaced culture as the current gold standard tests of choice for chlamydia and gonorrhea. The nontreponemal and treponemal serologic diagnostic and screening approach to syphilis has undergone less change. Treatment for gonorrhea includes a one-time dose of parenteral ceftriaxone and oral azithromycin. This treatment regimen not only addresses the possible gonococcal resistance seen in recent years but also treats chlamydia, which may present similarly or co-infect the same patient. Syphilis treatment in both pregnant women and their infected neonates remains with penicillin $\mathrm{G}$ formulations. It is crucial to establish close follow-up evaluation for the pregnant woman infected with these sexually transmitted infections to ensure adequate treatment and help prevent perinatal spread of the infection.
\end{abstract}

\section{Key Points}

Infection with chlamydia, gonorrhea, or syphilis can be asymptomatic or have a wide variety of clinical presentations in women who are pregnant or of childbearing age. If not identified or treated, these sexually transmitted infections can also infect fetuses and neonates.

Molecular-based tests are highly sensitive and specific and are therefore recommended for screening for chlamydia and gonorrhea. Screening for syphilis continues to be via serologic (nontreponemal and treponemal) testing.

Chlamydia can be treated with a one-time dose of oral azithromycin, gonorrhea can be treated with a one-time dose of parenteral ceftriaxone and oral azithromycin, and syphilis can be treated with a one-time dose of intramuscular benzathine penicillin $\mathrm{G}$ for primary infection or three weekly doses for secondary or tertiary stages of the infection.

Every case of congenital syphilis represents a missed opportunity for prevention by adequate screening, treatment, and follow-up of the pregnant woman.

Nizar F. Maraqa

nizar.maraqa@jax.ufl.edu

Pediatric Infectious Diseases, University of Florida College of Medicine Jacksonville, 841 Prudential Drive, Suite \# 1130, Jacksonville, FL 32207, USA

\section{Introduction}

The World Health Organization (WHO) estimates that more than 1 million sexually transmitted infections (STIs) are acquired every day worldwide, with an estimated 357 million new infections each year with one of the four principal STIs: chlamydia, gonorrhea, syphilis, and trichomoniasis [1]. As for the USA, the Centers for Disease Control and Prevention (CDC) estimates that 20 million new cases of STIs occur annually [2].

Screening for and treating STIs has not only significant implications for the acute health of the patient but also a significant role in preventing reproductive complications such as infertility or vertical transmission of infection to an offspring. STIs can have devastating implications on the health of the pregnant woman, her fetus, or the infant. For example, while syphilis can be mildly symptomatic in the pregnant woman, congenital infection may lead to stillbirth or present with severe clinical manifestations in the newborn, including hepatitis, pneumonitis, or neurosyphilis. As a result, numerous guidelines have been set forth by various organizations (e.g. WHO, CDC, US Preventive Services Task Force [USPSTF]) to address how, when, and who to screen for STIs [3-11].

The goal of this article is not to go over all these guidelines but to review the current knowledge about the management of the three main STIs (chlamydia, gonorrhea, 
and syphilis) in the context of pregnancy. We review the current epidemiology; key aspects of surveillance; the availability, sensitivity, and specificity of testing; treatment options; and issues related to linkage to care. We present to the reader a summary of these recommendations in Table 1.

\section{Chlamydia}

\subsection{Clinical Features}

Chlamydia trachomatis is an obligate intracellular bacterial pathogen with a range of clinical manifestations. In neonates and young infants, it can cause conjunctivitis, nasopharyngitis, and pneumonia. In these cases, the pathogen is primarily transmitted to the neonate via exposure to the mother's genital tract during vaginal birth. However, transmission has occurred following cesarean delivery with ruptured membranes and, more rarely, with intact membranes. The most common anatomical site infected in neonates is the nasopharynx, presenting as nasal stuffiness. Conjunctivitis may appear days to a few weeks after birth. In mild cases, it can lead to ocular congestion, edema, and discharge for 1-2 weeks, but it can also, rarely, lead to chronic infection causing local scarring and blindness. Pneumonia can present in the infant anywhere from 2 to 19 weeks after birth. It classically presents as staccato cough, tachypnea, and rales without fever. Infiltrates may be present on chest radiographs along with hyperinflation, but wheezing is uncommon. In older children and adults, C. trachomatis can lead to genitourinary tract infection or invasive lymphogranuloma venereum (LGV) [12].

In women, the cervix is the commonly infected site, and the urethra may be affected in a smaller proportion of women [13]. These cervical infections can ascend and cause pelvic inflammatory disease (PID), which can then lead to infertility, chronic pain, and ectopic pregnancy. Infection in pregnant women may cause adverse perinatal outcomes, including premature birth and transmission of the infection to the newborn. Most women with cervical infection will have no signs or symptoms to alert them to infection. In the National Longitudinal Study of Adolescent Health, 95\% of C. trachomatis-infected people were asymptomatic in the $24 \mathrm{~h}$ preceding specimen collection [14]. In addition, examination findings are abnormal in only the minority of women (about 10-20\% in some studies) [15]. When present, clinical signs include the "classic" cervicitis findings: mucopurulent endocervical discharge, endocervical friability, or edematous ectopy of the cervix. The incubation period ranges from 7 to 14 days following initial transmission. For asymptomatic women, the infection with chlamydia may be carried

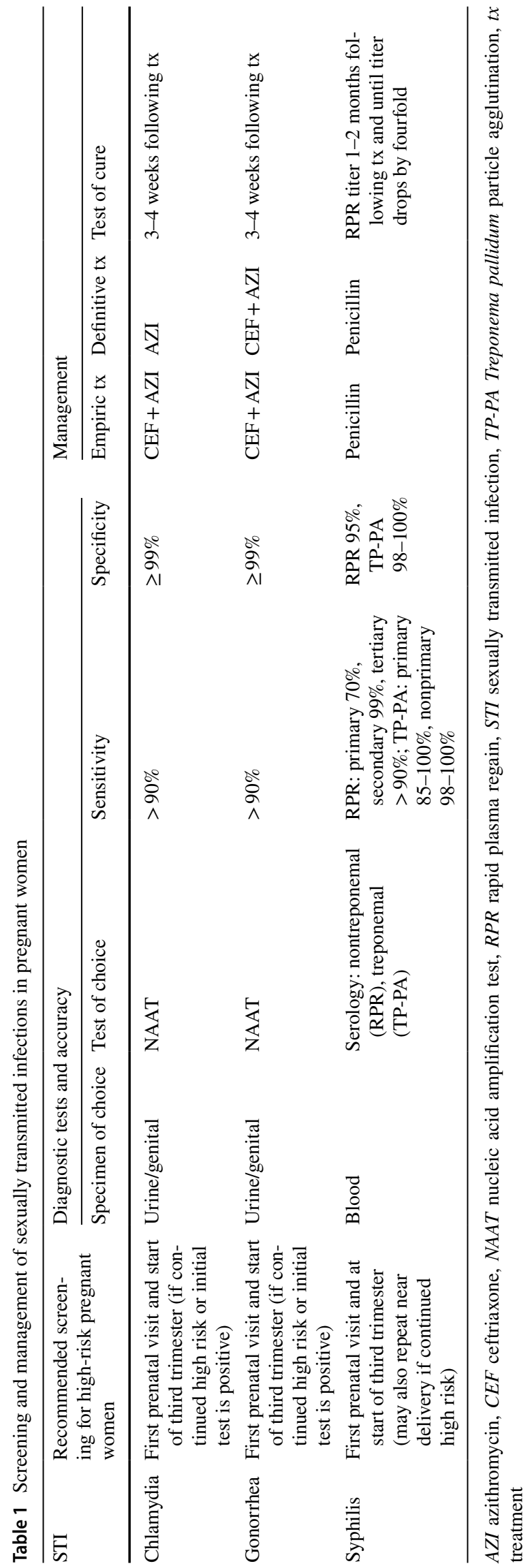


for several months to possibly a year, making it challenging to determine the infection date.

Chlamydia infection of the female urethra occurs in about $25 \%$ of those with cervical infection [13]. If symptoms are present, they are typical of urinary tract infection (UTI) with urinary frequency and dysuria. Urine analysis will show pyuria but no organisms on Gram stain or culture. This combination of pyuria with negative stain and culture should prompt the clinician to suspect chlamydial infection. C. trachomatis can also ascend to the upper reproductive tract and result in PID. This presentation will involve cervical motion tenderness (i.e. exquisite pain upon cervical manipulation with vaginal exam) along with abdominal or pelvic pain. The formation of adhesions may occur and is a major complication of PID. In about 5-15\% of PID cases, further ascension can lead to perihepatitis, with inflammation of the liver capsule and adjacent peritoneal surfaces (also known as Fitz-Hugh-Curtis syndrome). The patient usually experiences right upper-quadrant pain or pleuritic pain but has normal liver enzymes. PID can increase a woman's risk of infertility as well as ectopic pregnancy (due to inflammation and stricture of the fallopian tubes). Infection during pregnancy can increase the risk for premature rupture of the membranes, preterm delivery, spontaneous abortion, and stillbirth [16]. Reactive arthritis as well as its more serious presentation of Reiter's syndrome (arthritis, urethritis, and bilateral conjunctivitis) are complications that can occur with both $C$. trachomatis and Neisseria gonorrhoeae. This complication occurs more frequently in patients with the human leukocyte antigen (HLA)-B27 gene [17].

$\mathrm{LGV}$ is an invasive infection caused by $C$. trachomatis wherein the individual will develop genital ulcers and, in later stages, painful inguinal lymphadenopathy. It is caused specifically by L1, L2, and L3 serovars of C. trachomatis. In the past, LGV was typically rare in temperate climates. However, since 2003, it is increasingly being reported in developed countries such as the USA. After an incubation period of 3-12 days, the primary infection, consisting of a genital ulcer or other mucosal inflammatory reaction, occurs at the site of inoculation. These lesions will then spontaneously heal within a few days. The secondary stage appears about 2-6 weeks later and involves painful inflammation of the inguinal and/or femoral lymph nodes. Inflammatory masses may also develop in the rectum and retroperitoneum. If left untreated, late LGV can present with fibrosis and strictures in the anogenital tract [18].

\subsection{Epidemiology}

Chlamydia trachomatis is the most common reportable bacterial STI in the USA, with high rates among sexually active adolescents and young adult females. Most of those infected are asymptomatic and serve to provide an ongoing reservoir for transmission of the infection. According to the CDC, 1.59 million cases of chlamydia were reported in 2016, a $4.7 \%$ increase from 2015. Given the often-asymptomatic nature of chlamydia, it is estimated that the actual number of people infected annually is actually closer to 2.86 million. During 2014-2015, the CDC reported chlamydia infection rates of $1.5 \%$ among females aged $15-19$ years and $2.7 \%$ among young women aged $20-24$ years. It is estimated that 1 in 20 sexually active young women between 14 and 24 years of age has chlamydia [19].

Substantial racial/ethnic disparities exist with this pathogen. Non-Hispanic African Americans have 5.6 times the prevalence of non-Hispanic Caucasians. The settings of highest prevalence of chlamydia positivity have been family planning clinics $(8 \%)$, prenatal clinics $(7.2 \%)$, national job training programs (11.4\% in women, $7.2 \%$ in men), and adolescent correctional facilities $(14.5 \%$ in women, $6.5 \%$ in men) [20].

Chlamydia trachomatis presenting as conjunctivitis in infants was found at a rate of 2.1 cases per 100,000 live births in the USA from 2010 to 2015 [21]. In total, 50\% of infants born vaginally to infected mothers will acquire the pathogen. The risk of conjunctivitis is $20-50 \%$ in these infants, and the risk of pneumonia is 5-30\% [12].

\subsection{Diagnostic Tests}

Chlamydia trachomatis urogenital infections in females can be diagnosed via swab specimens collected from the cervix or vagina or via first catch (sometimes referred to as "dirty catch") urine collection. Nucleic acid amplification tests (NAATs) are the most sensitive (>90\%) and specific ( $\geq 99 \%$ ) for testing these specimens and, as such, are recommended for $C$. trachomatis detection. The sensitivity and specificity of older tests such as DNA probe, direct fluorescent antibody (DFA), and enzyme immunoassay (EIA) are inferior, and they are therefore not recommended for diagnosis of chlamydia urogenital infections.

The US FDA has approved several commercial NAATs for testing of vaginal, endocervical, and male intraurethral swabs and male/female urine specimens. These tests allow dual testing of specimens for both Chlamydia trachomatis and Neisseria gonorrhea, and some NAATs have been cleared for testing both clinician- and patient-collected vaginal samples in clinical settings. Individual NAAT package inserts should be reviewed, as the type of specimen they are approved to test may differ.

The FDA has not approved NAATs for testing rectal specimens for $C$. trachomatis. However, laboratories may perform this test if they have met Clinical Laboratory Improvement Amendments and other regulatory requirements and have validated their chlamydia NAAT 
performance on rectal swab specimens. NAAT detection of C. trachomatis from pharyngeal specimens collected from asymptomatic post-pubescent individuals is possible but not recommended, as clinical significance is unclear [22].

NAATs have not been approved for chlamydia testing on conjunctival, nasopharyngeal, tracheal aspirate, or lung biopsy specimens from infants as studies comparing them with culture are limited; however, they should theoretically be as superior to culture as for other specimens.

Testing is usually available at the local health department or other organizations that can perform these tests at no or low cost to those with minimal or no insurance coverage.

Both the USPSTF and CDC recommend screening for gonorrhea and chlamydia in high-risk pregnant women at the first prenatal visit. The CDC also recommends retesting at the third trimester in pregnant women with a continued risk for infection and those who tested positive at the first prenatal visit $[6,9,19]$. While age (14-25 years) remains an important risk factor for chlamydia infection, other women at high risk include those with new or multiple sex partners or a partner with an STI, those with inconsistent condom use, and those with a history of previous or coexisting STIs. No reliable studies have reported on the benefits or harms of screening asymptomatic pregnant women for chlamydia [5].

\subsection{Treatment}

Treatment for genital chlamydia should be started empirically, with coverage extended to also include gonorrhea, since co-infection may be present, and both may be asymptomatic or have similar symptoms and signs. When testing reveals the presence of only chlamydia, the treatment for children aged $\geq 8$ years, adolescents, or adults is oral doxycycline $100 \mathrm{mg}$ twice daily for 7 days or a single oral dose of azithromycin $1 \mathrm{~g}$ (available as pills or a single sachet to be mixed with a drink). Alternatives include oral erythromycin base $500 \mathrm{mg}$ four times daily for 7 days, erythromycin ethylsuccinate $800 \mathrm{mg}$ four times daily for 7 days, or doxycycline delayed release $200 \mathrm{mg}$ once daily for 7 days. Children infected with chlamydia aged $<8$ years may be treated with erythromycin $50 \mathrm{mg} / \mathrm{kg}$ per day, divided into four doses daily for 14 days when they weigh $<45 \mathrm{~kg}$ or with oral azithromycin $1 \mathrm{~g}$ in a single dose when they weigh $>45 \mathrm{~kg}$.

Azithromycin is currently recommended as the first-line choice for pregnant women, as studies suggest it is safe. Amoxicillin or erythromycin may be used as alternatives. Levofloxacin is another possible alternative that has been shown in human studies to be of low risk in pregnancy, although animal studies raise concerns of cartilage damage to neonates. Doxycycline is contraindicated during the second and third trimesters of pregnancy. While a test of cure is not routinely recommended for nonpregnant patients, it is recommended (preferably by NAAT) at 3-4 weeks after treatment for pregnant females as some of the regimens have not been as efficacious in that patient population [12].

For infected infants with either conjunctivitis or pneumonia due to $C$. trachomatis, the CDC and the American Academy of Pediatrics recommend treatment with oral erythromycin $50 \mathrm{mg} / \mathrm{kg}$ per day divided in four daily doses for 14 days. Topical therapy for conjunctivitis has proven not effective, has a high failure rate, and does not eradicate nasopharyngeal carriage. Infants should be followed closely, as they may need a second course of treatment if the symptoms do not resolve (in about $20 \%$ of cases). Oral azithromycin $20 \mathrm{mg} / \mathrm{kg}$ once daily for 3 days may be effective as an alternative agent. Parents should be educated about the possible increased risk of infantile hypertrophic pyloric stenosis in infants aged $<6$ weeks when treated with macrolides (particularly erythromycin) $[12,19]$.

The early detection and treatment of chlamydia infection in pregnant women and their sexual partners remains the best way to avoid infection in the infants. Reinfection is common in adolescents or adults after initial infection and treatment of chlamydia. Therefore, repeat testing for C. trachomatis is recommended 3 months following initial treatment or at the next healthcare visit, regardless of whether their sexual partner has received testing and/or treatment.

\section{Gonococcal Infections}

\subsection{Clinical Features}

Neisseria gonorrhea is a Gram-negative diplococcus that causes infections only in humans. Gonococcal infections tend to occur mostly in three distinct age groups: neonates, prepubertal children, and sexually active adolescents and young adults. For females in the latter age group, the infection is often asymptomatic. Clinical syndromes commonly include urethritis, endocervicitis, and salpingitis. In males, infection is often asymptomatic and primarily occurs in the urethra but can also occur in the rectum and pharynx. Infections in either sex can extend, resulting in epididymitis in males and bartholinitis, PID with potential tubal scarring and adhesions, and perihepatitis in females. Even asymptomatic gonococcal infections in females can progress to PID with tubal scarring, which can then lead to ectopic pregnancy, infertility, or chronic pelvic pain. Patients can also present with conjunctivitis, pharyngitis, or proctitis. In up to $3 \%$ of patients, gonococcal infections of mucosal sites may spread hematogenously to the skin and joints (causing arthritis-dermatitis syndrome or disseminated gonococcal infection). Patients 
with $N$. gonorrhea bacteremia can have a maculopapular rash (with or without necrosis), arthritis, and tenosynovitis [22].

The eye is the most common site of gonococcal infection in the newborn. The infection will typically present with purulent conjunctivitis, profuse exudate, and eyelid swelling. If not treated, this can then extend from the epithelial layers to the subconjunctival connective tissue, and to the cornea itself. Corneal involvement can lead to ulceration, scarring, and visual impairment. Perinatal gonococcal infections of this type most commonly present 2-5 days after birth. The application of ophthalmic antibiotic prophylaxis to the newborn, and other factors such as a low inoculum, may delay this presentation. Localized infection of other mucosal surfaces (e.g. pharynx, vagina, urethra, anus) can also occur [23]. Gonorrhea causing neonatal scalp abscess after placement of fetal monitoring electrode has been reported [24]. Infants infected with gonorrhea should be evaluated and treated for disseminated infection. Evaluation should include eye or conjunctival culture, blood culture, and cerebrospinal fluid (CSF) analysis and culture. Disseminated gonococcal disease may present as septic arthritis (most commonly), meningitis, or sepsis. Gonococcal arthritis in the neonate can present anywhere from 1 to 4 weeks of life, and multiple joints may be affected [25].

Gonococcal infection in prepubertal children may occur in the genital tract and is usually transmitted sexually. The most common presentation in prepubertal females is vaginitis. Infection may progress to cause PID, but this occurs less commonly than in adolescents. Males can develop urethritis, but this is uncommon. Anorectal and tonsillopharyngeal involvement can also occur and is usually asymptomatic [22].

\subsection{Epidemiology}

After chlamydia, gonorrhea is the second most commonly reported STI in the USA. In 2016, a total of 468,514 cases of gonorrhea were reported to the CDC, but it is estimated that approximately 820,000 cases occur each year in the USA alone. The CDC estimates place 570,000 of these cases among young people aged 15-24 years. The prevalence rates for females are 595.5 cases per 100,000 for those aged 20-24 years and 482.1 per 100,000 for those aged 25-29 years. As with chlamydial infection, there is racial disparity in the prevalence of gonorrhea, with 481.2 cases per 100,000 in African Americans, 242.9 per 100,000 in Native Americans, and 55.7 per 100,000 in Caucasians [26].

The rate of gonococcal ophthalmia in neonates in the USA from the years $2010-2015$ is reported to be $\leq 0.2$ cases per 100,000 live births per year [21].

\subsection{Diagnostic Tests}

Gonorrhea can be detected via microscopic examination of Gram-stained smears of exudate from the conjunctivae, vagina of prepubertal girls, male urethra, skin lesions, synovial fluid, and even CSF when clinically warranted. Identification of the Gram-negative diplococci inside white blood cells can also be helpful if the organism does not grow in culture. However, a negative smear is not considered sufficient to rule out gonococcal infection, as it tends to be of low sensitivity. For culture of $N$. gonorrhea, nonselective chocolate agar incubated in 5-10\% carbon dioxide is used for specimens from normally sterile sites (such as blood or CSF), whereas selective media that inhibit normal flora and nonpathogenic Neisseria organisms is needed for specimen from nonsterile sites (such as urethra, vagina, or rectum).

As with chlamydia, NAATs have superior sensitivity and specificity and are the tests of choice for $N$. gonorrhea detection regardless of specimen or test methodology (i.e. polymerase chain reaction [PCR], transcription-mediated amplification, or strand-displacement amplification). Most commercially available products are now approved by the FDA for testing male urethral swab specimens, female endocervical or vaginal swab specimens, male/female urine specimens, and liquid cytology specimens. The specimen is usually dually tested to detect both gonorrhea and chlamydia. Package inserts must be reviewed, as different tests have been approved for only specific specimen types.

The overall feasibility and compliance of patients and clinics with STI testing have risen with the increasing availability of less invasive testing. This is especially true with testing of male/female urine specimens and self-collected vaginal swabs. It must be remembered that urine for NAAT testing should be collected via first catch (or "dirty catch") specimen, whereas urine collected for other purposes (e.g. diagnosis of UTI) usually involves cleaning the urethra prior to collection. While such urethral cleaning may reduce the possibility of contamination by normal urogenital flora, it will kill any gonococcal bacteria and interfere with the detection of $N$. gonorrhea by NAAT. For screening purposes, the CDC recommends using a vaginal swab as the preferred NAAT testing source for females and urine for males (sensitivity $>90 \%$, specificity $\geq 99 \%$ ).

For non-genital sites, culture is the most widely used test. Several commercial laboratories have validated gonorrhea NAAT testing from rectal and/or pharyngeal sources. However, a risk of false-positive results exists for gonorrhea with some NAATs due to cross-reaction with nongonococcal Neisseria species in the throat [22]. 


\subsection{Treatment}

Treatment for gonorrhea genital infection is a single dose of intramuscular ceftriaxone $250 \mathrm{mg}$ combined with a single dose of oral azithromycin $1 \mathrm{~g}$ or oral doxycycline $100 \mathrm{mg}$ twice daily for 7 days. This regimen is also effective for treatment of chlamydia urogenital infection. While oral cefixime was an option for treatment when ceftriaxone was not available, this regimen is no longer recommended because of the increasing rates of gonococcal resistance to cefixime.

For pregnant women, the same treatment regimen with azithromycin and ceftriaxone is recommended. In cases of cephalosporin allergy or other contraindications to this treatment, consultation with an infectious disease specialist is recommended [22].

Infants with ophthalmia due to $N$. gonorrhoeae should be hospitalized and observed for response to therapy and risk of disseminated disease. Treatment should be started early upon clinical suspicion. While the presence of typical organisms in Gram staining of conjunctival specimen (which may later be confirmed by culture) is helpful to establish a definitive diagnosis, empirical treatment should not be withheld in neonates with a negative Gram stain and high maternal risk factors for gonorrhea. Treatment for infants with uncomplicated gonococcal ophthalmia neonatorum is a single dose of intravenous or intramuscular ceftriaxone $25-50 \mathrm{mg} / \mathrm{kg}$. A single dose of intravenous or intramuscular cefotaxime $100 \mathrm{mg} / \mathrm{kg}$ is an alternative option and is preferred for neonates with hyperbilirubinemia or receiving calcium-containing intravenous solutions. Ceftazidime is another alternative. Topical therapy is not recommended as it does not treat for possible systemic infection and is not needed when systemic therapy is used. In clinical practice, treatment is usually administered for $48-72 \mathrm{~h}$ or until systemic infection is ruled out and bacterial cultures are otherwise negative. If systemic or disseminated infection is present in the neonate, the duration of therapy is extended to at least 14 days [22].

\section{Syphilis}

\subsection{Clinical Features}

Syphilis is one of the important STIs to screen for and treat during pregnancy as intrauterine infection with the responsible agent, Treponema pallidum, can result in stillbirth, hydrops fetalis, or preterm birth. Infected infants may be born asymptomatic; however, they can go on to develop various manifestations classified as early (within the first 2 years of life) or late (after 2 years of age) congenital syphilis. Findings in early congenital syphilis include hepatosplenomegaly, copious nasal secretions (also known as "snuffles"), lymphadenopathy, mucocutaneous lesions, pneumonia, osteochondritis, pseudoparalysis, edema, rash, hemolytic anemia, and thrombocytopenia. These abnormalities typically present within the first 4-8 weeks of life. Untreated infants can develop late congenital syphilis, which usually involves the nervous system, bones and joints, eyes, teeth, and skin. Manifestations include interstitial keratitis (typically occurs at 5-20 years of age), eighth cranial nerve deafness (10-40 years of age), teeth malformations known as "Hutchinson teeth" and "mulberry molars", anterior bowing of the shins, frontal bossing, saddle nose deformity, perioral fissures known as rhagades, and symmetric swelling of the knees known as "Clutton joints".

Acquired syphilis in childhood, adolescence, and adulthood can be divided into three stages: primary, secondary, and tertiary syphilis. There is also a latent stage referred to as "latent syphilis", which can be divided into early and late phases. Primary syphilis appears as one or more painless raised ulcers of the skin or mucous membranes at the initial site of infection (oral or genital). These ulcers, known as chancres, usually appear around 3 weeks after exposure (range of 10-90 days) and spontaneously resolve without intervention within a few weeks. These lesions are painless and may go unrecognized in the clinical setting. However, they may still be present when the patient progresses to the secondary stage.

The secondary stage begins about 1-2 months later and is characterized by a rash that appears on the palms and soles. In addition, wart-like hypertrophic papular lesions, similar to those of human papillomavirus, can develop in moist areas around the vulva and anus; these are known as condyloma lata. Patients may also have generalized lymphadenopathy, fever, malaise, splenomegaly, sore throat, headache, arthralgia, and alopecia during this stage. The manifestations of secondary syphilis resolve after 3-12 weeks as the patient enters the latent stage, again becoming asymptomatic. During latent syphilis, patients are seroreactive but show no signs of disease. This latency may end within a year (early latent syphilis) or extend for longer than a year (late-latent syphilis).

Tertiary syphilis occurs around 15-30 years after the initial manifestations and can include growths of tissue around the body known as "gummas". Typically, the heart and aorta may become involved as well as the central nervous system (CNS). Involvement of the CNS, known as neurosyphilis, can include meningitis, uveitis, dementia, and posterior spinal cord degeneration. Of note, neurosyphilis can also present during any stage of syphilis infection. Patients living with HIV infection and neonates with congenital syphilis are especially prone to developing neurosyphilis [27].

\subsection{Epidemiology}

Syphilis is rare in most of the industrialized world but has persisted in the USA. In 2016, there were 27,814 cases of 
primary and secondary syphilis reported in the USA, a rate of 8.7 cases per 100,000 population. This was an increase of $17.6 \%$ since 2015 ( 7.4 cases per 100,000) and of $74.0 \%$ since 2012 (5.0 cases per 100,000) [28]. While syphilis rates in the USA are rising most prominently in men who have sex with men, the rate of primary and secondary syphilis in US women has also been increasing (from 1.1 cases per 100,000 in 2014 to 1.4 per 100,000 in 2015 and 1.9 per 100,000 in 2016) [28].

Syphilis is more common among pregnant women if they are poor, African American, young ( $<29$ years), and lack health insurance or prenatal care. Other risk factors include illicit drug use, co-infection with other STIs, residing in areas of high syphilis prevalence, and being a sex worker [29].

The rate of congenital syphilis in the USA increased by $86.9 \%$ between 2012 and 2016, with 15.7 cases per 100,000 live births reported in that year. Rates varied by maternal race/ethnicity, being highest for African American (43.1 cases per 100,000 live births), compared with Native American/Alaskan Native (31.6), Hispanic (20.5), Asian/Pacific Island (9.2), and Caucasian (5.3) women [28]. Most cases have occurred in southern USA and were mostly due to limited or late prenatal care. Importantly, many of these cases of congenital syphilis infection could have been prevented had maternal syphilis screening and management recommendations been followed correctly [27].

\subsection{Diagnostic Tests}

Definitive diagnosis of syphilis is made with identification of spirochetes on dark field examination of lesion exudate, nasal discharge, or tissue. T. pallidum can be detected by PCR, but no PCR assays have been cleared by the FDA for clinical diagnostic use. DFA tests are no longer available in the USA. A combination of nontreponemal and treponemal serologic tests are used to screen for and confirm the diagnosis of syphilis.

The nontreponemal tests used for syphilis diagnosis are the Venereal Disease Research Laboratory (VDRL) slide test and the rapid plasma reagin (RPR) test. Both these tests measure the level of antibody directed against a lipoidal antigen that is liberated with T. pallidum infection. They are inexpensive and can be performed rapidly. Lower titers from these tests are indicative of lower disease activity, usually because of treatment (usually indicated with a fourfold decrease in titer). The RPR and VDRL perform similarly, with sensitivity of $70 \%$ for primary, $99 \%$ for secondary, and $>90 \%$ for tertiary syphilis [30].

A false-negative nontreponemal test can occur in patients with early primary syphilis, long duration of latent acquired syphilis, and late congenital syphilis. A falsenegative (or weakly reactive) nontreponemal test may also occur because of the "prozone" phenomenon, where the very high antibody levels against $T$. pallidum bind to the antigen, thus preventing the test from detecting it. Diluting the patient's serum will correct this prozone effect and provide a true-positive result.

These antibodies to the lipoidal antigen are also produced in a variety of conditions unrelated to syphilis. The incidence of false-positive nontreponemal tests is generally only about $1-2 \%$. Causes include advancing age, endocarditis, phospholipid antibody syndrome, systemic lupus erythematosus, rheumatoid arthritis, idiopathic thrombocytopenic purpura, malignancy, injection drug use, chronic liver disease, and strong acute viral or bacterial infection. Usually about $90 \%$ of false-positive reactions have a titer of $<1: 8$. Chronic false-positive reactions that persist for more than 6 months are often associated with autoimmune disorders and chronic inflammation [30, 31].

RPR titers are typically higher than VDRL titers; therefore, when monitoring response to treatment, the same test must be used, preferably performed by the same laboratory. Response to treatment is seen as a fourfold decrease in titer or change in two dilutions (e.g. 1:64 to $1: 16$ ), whereas the opposite can be indicative of reinfection or relapse. Typically, the RPR is used to test serum samples because a stabilized antigen can be used, whereas the VDRL needs daily preparation of fresh antigen suspension. The VDRL is mainly used to test CSF when indicated, as the RPR test is less sensitive and specific for CSF testing and is therefore not recommended.

Treponemal tests include the T. pallidum particle agglutination (TP-PA) test, the fluorescent treponemal antibody absorption (FTA-ABS) test (both of which are used as confirmatory tests in the "conventional diagnostic" approach), and the T. pallidum EIA (TP-EIA) and T. pallidum chemiluminescent assay (TP-CIA) (used as initial screening tests in the "reverse-sequence screening" approach). A positive treponemal test in patients with syphilis typically remains positive for life, even after successful therapy. However, $15-25 \%$ of patients treated during the primary stage revert to a seronegative state after 2-3 years. Even though treponemal tests are more specific than nontreponemal tests for syphilis, false-positive treponemal results may be seen with other spirochetal infections such as Lyme disease (borreliosis) or leptospirosis.

The CDC and USPSTF traditionally recommended conventional screening first with the nontreponemal tests (RPR, VDRL), then confirming with treponemal-specific tests (TP-PA or FTA-ABS) to help rule out nontreponemal causes of RPR reactivity. Some laboratories have started using a "reverse-sequence screening" strategy as an alternative to conventional testing by performing a screening treponemal antibody test (TP-CIA or TP-EIA) first, then the nontreponemal test to save costs and labor. However, 
this reverse algorithm may have unacceptably high rates of false-positive results when used in low-prevalence populations [32]. When the reverse-sequence algorithm is used, people with a positive TP-EIA/TP-CIA result and a negative nontreponemal test result (discordant result) should undergo a second treponemal test targeting a different $T$. pallidum antigen to confirm the results of the original test. If the second treponemal test result is negative and the person is at low risk for syphilis, the original treponemal test result was likely false positive [27].

It is recommended that pregnant women are screened for syphilis early in pregnancy and that testing is repeated at delivery. In settings with a high prevalence of syphilis, testing early in pregnancy, at the beginning of the third trimester ( $\sim 28$ weeks of gestation), and at delivery is recommended. For women who test positive and are treated, follow-up serologic testing is necessary to determine effectiveness of therapy.

Occasionally, pregnant women can develop low-titer, false-positive nontreponemal antibody tests. Hence, all positive results should be confirmed with treponemal tests. Usually, a negative confirmatory treponemal antibody test confirms that a positive nontreponemal test was a false positive. A rare exception could occur in patients with early syphilis infection, in whom the nontreponemal test becomes positive before the treponemal test. Therefore, repeat testing in 2-4 weeks, and as clinically indicated, is recommended.

The sensitivity and specificity of treponemal serologic tests for syphilis is high. The FTA-ABS test has a sensitivity of $84 \%$ for detecting primary syphilis and almost $100 \%$ for detecting syphilis infection in other stages, and its specificity reaches $96 \%$ [33]. For the TP-PA test, primary syphilis is detected with a sensitivity of $85-100 \%$ and a specificity of 98-100\%. For secondary and late-latent syphilis, the TP-PA has a sensitivity of $98-100 \%$ and specificity of $98-100 \%$ [34].

\subsection{Treatment}

The "gold standard" treatment for syphilis in both pregnant and nonpregnant females is penicillin $\mathrm{G}$, as no clinically relevant penicillin-resistant strains of $T$. pallidum have been identified. Penicillin treatment of syphilis during pregnancy has been shown to be effective for treating maternal disease, preventing vertical transmission to the child, and treating any established fetal disease. Syphilis treatment guidelines are based on disease staging, as later stages of infection require longer duration of therapy because of the slower rate of treponemal replication [19]. For primary, secondary, and early latent syphilis, a single intramuscular dose of benzathine penicillin $\mathrm{G}$ at 50,000 units $/ \mathrm{kg}$ (up to a maximum dose of 2.4 million units) is recommended. For late-latent syphilis or syphilis of unknown duration, three intramuscular doses of 50,000 units/kg (up to 2.4 million units per dose) are recommended at 1 -week intervals. For patients who are allergic to penicillin and not pregnant, oral doxycycline $100 \mathrm{mg}$ twice daily or tetracycline $500 \mathrm{mg}$ four times daily for 14 days is an acceptable alternative. It is recommended that pregnant women with syphilis who are allergic to penicillin be desensitized and still treated with penicillin. The same applies to neurosyphilis and congenital syphilis, where penicillin is the only recommended therapy.

For proven or probable congenital syphilis (based on physical examination of the infant, laboratory test results from both mother and infant, and adequacy of maternal treatment for syphilis), the preferred treatment is intravenous aqueous crystalline penicillin $\mathrm{G}$ for 10 days. The recommended dose is 50,000 units/kg every $12 \mathrm{~h}$ for the first 7 days of life and the same dose every $8 \mathrm{~h}$ thereafter. Alternatively, intramuscular procaine penicillin G 50,000 units/kg can be administered as a single daily dose for 10 days. This latter regimen is not recommended for treatment of neurosyphilis as it achieves low CSF concentrations. If more than 1 day of therapy is missed, the entire course should be restarted. Data are inadequate to support the use of other antimicrobial agents (e.g. ampicillin) for the treatment of congenital syphilis. After treatment, serological nontreponemal testing should be obtained every 2-3 months until the test becomes nonreactive or the titer has decreased at least fourfold. Nontreponemal titers should decrease by 3 months of age and be nonreactive by 6 months of age with adequate treatment. For congenital neurosyphilis, a repeat CSF examination and VDRL testing at the age of 6 months is also recommended to document adequacy of treatment. If the child has increasing titers or persistent stable titers 6-12 months after treatment, repeat evaluation is recommended (including CSF examination) as well as a repeat 10-day course of parenteral penicillin G [27].

\section{Conclusion}

The burden of STIs on our present population and future generations is immense. Screening pregnant women for these infections is not only important for the health of the woman but can also be especially critical to the wellbeing of her fetus and the health of her newly born infant.

As new, rapid, less invasive, easy-to-collect and more accurate tests become readily available, providers have more opportunities to detect, treat, and prevent transmission of these infections during pregnancy. Healthcare providers should keep abreast of the most current national recommendations and guidelines for screening and management of STIs. Future research and scientific advances will surely continue to reduce the impact of these STIs on pregnant women and their offspring. 


\section{Compliance with Ethical Standards}

Conflict of interest Harold J. Lochner III and Nizar F. Maraqa have no conflicts of interest.

Funding No sources of funding were used to support the writing of this manuscript.

\section{References}

1. World Health Organization. Media centre-fact sheets: sexually transmitted infections (STIs). Updated August 2016. http://www. who.int/mediacentre/factsheets/fs110/en/. Accessed 26 Apr 2018.

2. National Center for HIV/AIDS, Viral Hepatitis, STD, and TB Prevention (U.S.). Division of STD Prevention. Centers for Disease Control and Prevention. CDC Fact Sheet. Reported STDs in the United States: 2014 national data for chlamydia, gonorrhea, and syphilis. 2015. https://stacks.cdc.gov/view/cdc/36834. Accessed 26 Apr 2018.

3. World Health Organization. Global strategy for the prevention and control of sexually transmitted infections: 2006-2015: breaking the chain of transmission. World Health Organization/Publications/Sexual and Reproductive Health/Sexually Transmitted and Reproductive Infections. http://www.who.int/reproductivehealth/ publications/rtis/9789241563475/en/. Accessed 26 Apr 2018.

4. Workowski KA, Bolan GA, Centers for Disease Control and Prevention. Sexually transmitted diseases treatment guidelines, 2015. MMWR Recomm Rep. 2015;64(RR-03):1-137.

5. LeFevre ML. Screening for chlamydia and gonorrhea: U.S. Preventive Services Task Force recommendation statement. Ann Intern Med. 2014;161(12):902-10. https://doi.org/10.7326/ M14-1981.

6. U.S. Preventive Services Task Force. Screening for chlamydial infection: U.S. Preventive Services Task Force recommendation statement. Ann Intern Med. 2007;147(2):128-34.

7. U.S. Preventive Services Task Force. Screening for gonorrhea: recommendation statement. Ann Fam Med. 2005;3:263-7.

8. U.S. Preventive Services Task Force. Screening for syphilis infection in pregnancy: U.S. Preventative Services Task Force reaffirmation recommendation statement. Ann Intern Med. 2009;150(10):705-9.

9. Final Recommendation Statement. Chlamydia and gonorrhea: screening. U.S. Preventive Services Task Force. 2016. https:// www.uspreventiveservicestaskforce.org/Page/Document/Recom mendationStatementFinal/chlamydia-and-gonorrhea-screening. Accessed 26 Apr 2018.

10. American College of Obstetrics and Gynecology. Annual women's healthcare. https://www.acog.org/About-ACOG/ACOG-Depar tments/Annual-Womens-Health-Care/FOR-PATIENTS/Pt-Exams -and-Screening-Tests-Age-19-39-Years. Accessed 26 Apr 2018.

11. Expedited partner therapy in the management of gonorrhea and chlamydial infection. Committee Opinion No. 632. American College of Obstetricians and Gynecologists. Obstet Gynecol 2015;125:1526-28.

12. American Academy of Pediatrics. Chlamydia trachomatis. In: Kimberlin DW, Brady MT, Jackson MA, Long SS, editors. Red Book ${ }^{\circledR}: 2018$ report of the committee on infectious diseases. Itasca: American Academy of Pediatrics; 2018. p. 276-83.

13. Stamm WE. Chlamydia trachomatis infections of the adult. In: Holmes KK, Sparling PF, Mardh PA, et al., editors. Sexually transmitted diseases. 4th ed. New York: McGraw-Hill; 2008. p. 575-94.
14. Miller WC, Ford CA, Morris M, et al. Prevalence of chlamydial and gonococcal infections among young adults in the United States. JAMA. 2004;291:2229-36.

15. Marrazzo JM. Mucopurulent cervicitis: no longer ignored, but still misunderstood. Infect Dis Clin N Am. 2005;19(2):333-49.

16. Darville T, Rours IJG. Chlamydia trachomatis. In: Long SS, Prober CG, Fisher M, editors. principles and practice of pediatric infectious diseases. 5th ed. New York: Elsevier; 2017. p. 908-14.

17. Hong DK, Gutierrez K. Infectious and inflammatory arthritis. In: Long SS, Prober CG, Fisher M, editors. Principles and practice of pediatric infectious diseases. 5th ed. New York: Elsevier; 2017. p. 487-93.

18. Mabey D, Peeling RW. Lymphogranuloma venereum. Sex Trans Infect. 2002;78(2):90-2.

19. Centers for Disease Control and Prevention. Sexually transmitted disease surveillance 2015. STDs in adolescents and young adults. Archived October 17, 2016. https://www.cdc.gov/std/stats15/adole scents.htm. Accessed 26 Apr 2018.

20. Centers for Disease Control and Prevention. Sexually transmitted disease surveillance 2010. Atlanta: U.S. Department of Health and Human Services; 2011.

21. Kreisel K, Weston E, Braxton J, Llata E, Torrone E. Keeping an eye on chlamydia and gonorrhea conjunctivitis in infants in the Unites States, 2010-2015. Sex Transm Dis. 2017;44(6):385-6.

22. American Academy of Pediatrics. Gonococcal Infections. In: Kimberlin DW, Brady MT, Jackson MA, Long SS, editors. Red Book ${ }^{\circledR}: 2018$ report of the committee on infectious diseases. Itasca: American Academy of Pediatrics; 2018. p. 355-65.

23. Darville T. Gonorrhea. Pediatr Rev. 1999;20(4):125-8.

24. Varady E, Nsanze H, Slattery T. Gonococcal scalp abscess in a neonate delivered by caesarean section. Sex Transm Infect. 1998;74(6):451.

25. Babl FE, Ram S, Barnett ED, Rhein L, Carr E, Cooper ER. Neonatal gonococcal arthritis after negative prenatal screening and despite conjunctival prophylaxis. Pediatr Infect Dis J. 2000;19(4):346-9.

26. Centers for Disease Control and Prevention. 2016 Sexually transmitted diseases surveillance: gonorrhea. Updated September 26, 2017. https://www.cdc.gov/std/stats16/gonorrhea.htm. Accessed 26 Apr 2018.

27. American Academy of Pediatrics. Syphilis. In: Kimberlin DW, Brady MT, Jackson MA, Long SS, editors. Red Book®: 2018 report of the committee on infectious diseases. Itasca: American Academy of Pediatrics; 2018. p. 773-88.

28. Centers for Disease Control and Prevention. 2016 sexually transmitted diseases surveillance: syphilis. Updated September 26, 2017. https://www.cdc.gov/std/stats16/Syphilis.htm. Accessed 26 Apr 2018.

29. Rac MW, Revell PA, Eppes CS. Syphilis during pregnancy: a preventable threat to maternal-fetal health. Am J Obstet Gynecol. 2017;216(4):352-63.

30. Cadavid D. Spirochetal infections. Handb Clin Neurol. 2010;96:179-219.

31. Ratnam S. The laboratory diagnosis of syphilis. Can J Infect Dis Med Microbiol. 2005;16(1):45-51.

32. Soreng K, Levy R, Fakile Y. Serologic testing for syphilis: benefits and challenges of a reverse algorithm. Clin Microbiol Newsl. 2014;36(24):195-202.

33. Golden MR, Marra CM, Holmes KK. Update on syphilis: resurgence of an old problem. JAMA. 2003;290:1510-4.

34. Creegan L, Bauer HM, Samuel MC, et al. An evaluation of the relative sensitivities of the venereal disease research laboratory test and the Treponema pallidum particle agglutination test among patients diagnosed with primary syphilis. Sex Transm Dis. 2007:34:1016-108. 